\title{
SALIVA APPLICATION IN ORAL AND SYSTEMIC DISEASES
}

\author{
Mariana Yordanova ${ }^{1}$, Daniela Gerova ${ }^{1}$, Bistra Galunska ${ }^{2}$ \\ ${ }^{1}$ Department of General Medicine and Clinical Laboratory, \\ Faculty of Medicine, Medical University of Varna \\ ${ }^{2}$ Department of Biochemistry, Molecular Medicine and Nutrigenomics, \\ Faculty of Pharmacy, Medical University of Varna
}

\begin{abstract}
Saliva is a noninvasive and accessible biofluid that permits early detection of oral and systemic diseases. Changes in saliva reflect the alterations in the blood and thus making saliva a suitable diagnostic tool. The use of saliva has many advantages, including simple and non-invasive sampling and easy, low-cost storage.

Today salivary diagnostics is a promising tool for diagnostic processes and clinical monitoring. Saliva is used to detect illicit drugs, alcohol, to measure hormone levels, and in the diagnosis of wide range systemic diseases such as cardiovascular, infectious, renal, endocrine diseases, some types of cancer as well as nonsystemic oral diseases.

The current review presents a critical overview of saliva as a promising tool for the development of valuable salivary biomarkers, their relevance to the prognosis, diagnosis and management of systemic and oral diseases. Development of point-of-care testing based on saliva samples as a screening tool will also support the diagnostic process in near future.
\end{abstract}

Keywords: saliva, salivary biomarkers, systemic and oral diseases

\section{INTRODUCTION}

Saliva is an aqueous slightly acidic ( $\mathrm{pH}$ 6-7) fluid of the oral cavity secreted by several types of salivary glands. The salivary secretions differ considerably in their composition and are affected by different physiologic and pathologic factors such as time of the day, dietary regimen, age, gender, system or oral cavity diseases, and drugs (1). The composition of saliva is very complex comprising different in their nature secretory products (organic and inorganic).

Address for correspondence:

Mariana Yordanova

Faculty of Medicine

Medical University of Varna

55 Marin Drinov St

9002 Varna

e-mail:m.jordanova.doc@abv.bg

Received: April 1, 2018

Accepted: June 29, 2018
These salivary components mostly are ingredients of salivary glands secretion, oropharynx and upper airway fluids, gastrointestinal reflux fluid, gingival sulcus fluid, food deposits in the oral cavity, and bloodderived compounds.

As a body fluid, saliva plays a very complex biologic role and supplies a large range of physiologic functions. In the oral cavity, saliva is important for mastication, normal speech and deglutition function, gustatory sensitivity, oral cavity lubrication and mucosal protection against microbial invasion. It takes place in post-eruptive maturation and carioprotection. In the gastrointestinal tract the saliva is important for esophageal physiology, enhances the digestive process, and contributes to mucosal protection (2).

\section{Salivary Composition}

Healthy adult subjects normally produce 500 $1500 \mathrm{~mL}$ of saliva per day, at a rate of approximately $0.5 \mathrm{~mL} / \mathrm{min}$. Salivary composition and volume can 
be affected by various physiological and pathological factors including olfactory and gustatory factors, chewing process, psychological and hormonal status, and drugs. Additionally the age, heredity, oral hygiene and physical exercise can also contribute to the salivation process (3).

Being an aqueous fluid, the saliva is composed of more than $99 \%$ water. The inorganic and organic part of the saliva reveals wide inter- and intraindividual variations. The inorganic matter is composed mostly of $\mathrm{Na}^{+}, \mathrm{K}^{+}, \mathrm{Cl}, \mathrm{Ca}^{2+}, \mathrm{HCO}_{3}^{-}, \mathrm{Mg}^{2+}$, and $\mathrm{NH}_{3}$. The organic portion is represented by urea, uric acid, creatinine, biogenic amines (putrescine, cadaverine), lipids such as cholesterol and fatty acids, and more than 400 types of proteins. Most of the proteins are of glandular origin (a-amylase, histatins, cystatins, lactoferrins, lysozymes, mucins, and proline-rich proteins (PRPs)) or plasma origin (albumin, secretory immunoglobulin A, transferrin) (2).

There is a strong relationship between the composition and the functions of saliva. For example, the a-amylase, mucins and PRPs are related to digestive, mastication and deglutition function, lactoferrins, lysozymes, and sIgA contribute to the protective and defense function.

\section{Collecting Saliva and Sample Handling}

The technique of saliva sample taking is one of the most important sources of errors affecting the final result. Two aspects have to be taken into account: the type of saliva - whole or gland-specific, and the level of secretion stimulation - stimulated or nonstimulated.

Studies of oral pathology, salivary gland pathology and systematic diseases most frequently use whole saliva, containing fluid from both local and systemic sources. Collection of whole saliva samples is a simple procedure and requires minimal equipment (4). Passive drool is a saliva collection method for collecting whole saliva (also called "mixed" saliva). Passive drool is considered to be the gold standard when collecting saliva samples for biological testing, because it provides the purest sample possible and allows researchers to "biobank" samples for future testing. The method ensures rapid sample collection and sample integrity.

There are also established methods for taking glandular saliva samples. Ductal and parotid secre- tion collection is usually done using sterile-modified Carlson-Crittenden/Lashley cup fitted with appropriate polyvinyl chlorate tubing. This device requires suction, which can be provided by a dental unit suction, laboratory suction bulb, or oil-free portable vacuum pump. Submandibular and sublingual secretions can be collected using submandibular and sublingual saliva collector fitted with a sterile $100 \mu \mathrm{L}$ pipette tip and a low-affinity plastic conical collection tube. This device requires suction, which can be provided by a dental unit suction or oil-free portable vacuum pump (5).

The composition of saliva depends on stimulation: nonstimulated saliva is considered to be a neutral sample, less-affected by salivary glands, while stimulated saliva is recommended for a more precise detection of cancer biomarkers. A very important issue is the standardization of saliva sample collection procedure by taking into account the saliva circadian rhythms and by removing oral stimulants such as eating, drinking and oral hygiene practices before sample collection (4).

Immediate processing of the sample is recommended in order to avoid decomposition, where this is not possible, the sample may be stored at $-80^{\circ} \mathrm{C}$. It is important to clean and refine the saliva removing desquamated epithelial cells, microorganisms and remnants of food (4).

\section{Using Saliva as a Diagnostic Fluid}

Saliva has been used in diagnostics for many years. In ancient traditional Chinese medicine saliva and blood are considered 'brothers' with a common origin and changes in saliva composition are indicative for the patient's wellness (1).

It is well known that a thin layer of epithelial cells separates the salivary ducts from the systemic circulation making possible the exchange of substances between saliva and blood plasma by active transport, ultrafiltration or passive membrane diffusion. Therefore, the changes in saliva reflect the alterations in the blood and thus making saliva a suitable diagnostic tool (2).

As a diagnostic tool, saliva provides some advantages over blood serum/plasma related to noninvasive and simple sample collection not requiring additives such as anticoagulants. Saliva samples 
are preferable for diagnostics for patients at risk for blood sampling such as hemophiliacs (6).

Nowadays saliva samples are used in therapeutic drug monitoring, doping control, steroidal hormones analysis, and in the diagnostics of infection diseases. Saliva can potentially be applied in the diagnosis of autoimmune, cardiovascular, renal, and oral diseases, biomarkers testing for malignancy, forensic purposes, and psychological research (7).

The usage of saliva as a diagnostic tool is an analytical challenge related to high variability of bioactive compounds in saliva samples and lack of established reference values for most of the routine laboratory parameters. For each tested systemic biomarker a high correlation between saliva and plasma should be established. All components produced locally by the salivary glands, such as sIgA and lysozyme, reveal low plasma levels and can be tested predominantly in the saliva (3).

\section{Infection}

It is considered that saliva is an useful alternative diagnostic tool for numerous infectious diseases such as viral hepatitis, tuberculosis, and Helicobacter pylori (HP) infection.

As a diagnostic test saliva can be used for detection of bacterial and viral pathogens. Usually a combined approach is used measuring both antibody and antigen or antibody and nucleic acid in saliva. This approach is based on the fact that saliva contains a wide spectrum of molecules including immunoglobulins (Ig), and nucleic acids originating from wide range viral and microbial pathogens (8).

In the course of hepatitis viral infection IgM, $\operatorname{IgG}$, and IgA antibodies are detectable not only in serum, but also in saliva. Due to their simplicity of sample collection, saliva tests have been reported as an alternative to conventional serum testing for hepatitis viral infection. However, the sensitivity of detecting hepatitis viral antigens in saliva is 1 to $3 \log 10$ units lower than that in serum (9).

Therefore, a quantitative DNA/RNA analysis is nowadays used for evaluation the hepatitis virus infection $(6,10)$

Quantitative DNA analysis is also used for testing Mycobacterium tuberculosis in the saliva. The analysis is usually done by the polymerase chain re- action during the acute phase of the disease when the bacterial load is high.

Saliva is also used in the diagnosis of HP infection as the oral cavity may be a source of infection (4). HP is related to a multitude of pathologies such as atrophic gastritis, gastric and duodenal ulcers. HP is a Gram-negative, microaerophilic bacterium which plays an important role in the natural stomach ecology. As it binds to salivary mucins MUC-5B and MUC 7 , secreted by the mucous and serous acinar cells of the seromucous salivary glands, higher salivary levels of these mucins could be used as indicative biomarkers for HP infection (8).

\section{Malignancy}

Salivary analysis may aid in the early detection and screening of certain malignant tumors. Saliva also aids in monitoring the efficacy of treatment. The well-known serum marker for breast cancer 15-3 (CA15-3) can also be detected in saliva. Increased levels of CA15-3 were established in the saliva of women diagnosed with breast cancer compared to controls. In addition, the tumor marker c-erbB-2 (erb), considered as more robust biomarker of breast cancer than CA15-3, was detected in the saliva of breast cancer patients and absent in controls (11). Another tumor marker found to be elevated in the saliva of women with active and nonactive breast cancer is the epidermal growth factor.

The cancer antigen CA125 routinely used as a serum marker of ovarian cancer, was found to be also elevated in the saliva of ovarian cancer patients. It is considered that salivary CA125 levels are a better diagnostic marker of the disease than the serum values $(12,13)$.

The tumor suppressor protein $\mathrm{p} 53$ is produced in a response to various types of DNA damages. Inactivation of p53 by mutations or gene deletions is frequently related to cancer development. Antibodies against mutated p53 have been suggested as possible markers for early diagnosis of oral squamous cell carcinoma (OSCC) (14). The p53 antibodies can be detected in the saliva of patients diagnosed with oral squamous cell carcinoma (1). In a recent study, a significantly elevated salivary levels of anti-p53 were established in OSCC patients compared to healthy controls. Anti-p53 in saliva can also be used as an early marker for OSCC (15). 


\section{Cardiovascular Disease (CVD)}

Early diagnosis and early intervention is the key for a good CVD patients' prognosis especially in emergent cardiovascular incidents. Saliva is considered as an ideal fluid for a non-invasive and rapid testing with needed analytical and clinical reliability that could be used to minimize the time from diagnosis to the treatment. The nano-bio-chips (NBC) are specific type of point-of-care devices for the needs of early diagnosis, periodic monitoring, and treatment of disease. The new and sensitive NBCbased analytical devices are an excellent tool to overcome the main drawbacks of salivary analysis such as low specificity and sensitivity. NBC-based system is developed for detection of salivary CRP based on antigen and $\mathrm{Ab}$ interactions (16). Additionally, a multiplex platform "cardiac arrest rapid diagnostic information using saliva" (CARDIUS) is developed for acute myocardial infarction screening, using antibodies against myoglobin, C-reactive protein, myeloperoxidase and IL-1 $\beta$ (17). A disposable electrochemiluminescent biosensor was developed for the analysis of lactate, marker for acute myocardial infarction, using lactate oxidase and luminol based detection system (16.)

For pre- and post-operative control of CVD patients, salivary alfa-amylase is used as a biomarker. A study of Adam et al. showed that low levels of salivary amylase in the preoperative stage of aorta aneurysm are associated with increased mortality (18). Another salivary enzyme reported to be associated with hypertension, a major risk factor for cardiovascular disorders is lysozyme (6).

\section{Renal Diseases}

Numerous salivary ingredients including cortisol, nitrite, uric acid, sodium, chloride, $\mathrm{pH}$, amylase and lactoferrin are considered to be associated with end-stage renal disease and developed as salivary biomarkers $(19,20)$. A colorimetric salivary test strips for nitrate and uric acid are developed for the establishment of the hemodialysis regimen. Salivary phosphate has been successfully used as a clinical biomarker for hyperphosphatemia related to cardiovascular complications in chronic renal failure patients (21).

\section{Monitoring Hormone Levels}

Salivary analysis can be used as part of endocrine function evaluation (22). The steroid hor- mones, being lipophilic, are usually transported in the blood bound to specific proteins such as SHBG (sex hormone-binding globulin) and CBG (cortisolbinding globulin) and to a lesser extent - to albumin. The unbound fraction, $1-2 \%$ of their total content, is considered bioactive, responsible for their physiologic effects. The salivary steroids are mainly in an unbound form. They reflect the plasma concentrations of bioactive hormones as the steroid hormone binding proteins are not found in saliva and salivary albumin concentration is more than 20 times lower than that in plasma (23). Currently saliva is used for determination of aldosterone, cortisol, cortisone, dehydroepiandrosterone, estradiol, estriol, progesterone, and testosterone levels. Monitoring salivary testosterone levels may be useful in behavioral studies of aggression, depression, abuse, violent and antisocial behavior $(1,3)$.

\section{Drug Monitoring}

Saliva is a convenient noninvasive tool for screening substance dependency. Currently, saliva is used for qualitative analysis of nicotine, cannabinoids, cocaine, phencyclidine, lysergic acid diethylamide, marijuana, opioids, barbiturates, diazepines, amphetamines and ethanol. Express salivabased tests are developed for detection of the most common drugs of abuse and ethanol. These tests are used by law enforcement agencies for roadside evaluation of alcohol and abuse drug testing and in hospital emergency departments as a rapid means of determining whether impaired consciousness is related to alcohol intoxication.

Salivary nicotine testing is useful in monitoring self-reported compliance with smoking cessation programs (1).

\section{Forensic Evidence}

Saliva is also a useful source for biomarker profiling and forensic identification. It can be used in bite mark gender determination and in animal bite mark analysis (24). DNA and mRNA can be extracted from bite marks, cigarette butts, postage stamps, envelopes and other objects, as during the biting process, saliva is deposited on the skin or on the object surface in enough amount to allow DNA recovering and consequent PCR analysis (6). 
Mariana Yordanova, Daniela Gerova, Bistra Galunska

\section{Oral Diseases}

Dental caries, saliva secretion rate and buffering capacity have proven to be sensitive parameters in caries prediction models. High numbers of $S$. mutans and Lactobacillus indicate a shift in oral microflora from healthy to more cariogenic. Diagnostic kits for S. mutans and Lactobacillus counting and salivary buffering capacity are widely used in dental practice and can be conducted without laboratory facilities (6).

Periodontitis, a chronic, inflammatory disease destructing the supporting tissues of the teeth is provoked by pathogenic periodontal microorganisms found in the plaque biofilm and around gingival margins of the teeth (25). Traditionally a plaque or pocket samples are used for either anaerobic culturing or DNA-PCR analysis. Ongoing research has shown that saliva reveals almost identical results to those obtained by plaque or pocket sampling.

Another field of salivary diagnostics is oral cancer, increasing nowadays dramatically. Recent studies have shown that squamous cell carcinoma comprises 3\% of all malignancies diagnosed in the United States, making it the tenth most common malignancy (26). Salivary RNA biomarkers yield 91\% sensitivity and specificity in distinguishing oral squamous cell carcinoma from controls $(27,28)$.

It has been shown that local chronic oral inflammation processes may influence various systemic diseases such as anemia, gastritis, colitis, atherosclerosis and vascular diseases (29). Plaque biofilm releases a variety of biologically active products such as endotoxins, cytokines and protein toxins from Gram-positive and Gram-negative bacteria colonizing the tooth surface around the gingival margin and interproximal areas. These molecules penetrate the gingival epithelium and initiate a host response. As a result the gingival epithelium produces chemical mediators including interleukin-1 beta (IL-1), prostaglandins, tumor necrosis factor alpha (TNF- $\alpha$ ), and matrix metalloproteinases. These products function as attractive molecules for the recruitment of neutrophils, T-cells, and monocytes. Antibodies specific to oral bacteria circulate in the peripheral blood and the acute-phase response becomes activated, CRP, fibrinogen and complement are produced both by lo- cal cells and within the liver. These proteins may further exacerbate various systemic diseases $(30,31)$.

\section{CONCLUSION}

The ongoing development of salivary diagnostics and the ease of collection of saliva shift the paradigm in diagnostic and treatment approaches in many areas of medicine and dentistry. Saliva is a useful specimen especially when a qualitative answer is required. It can also be used for quantification of different analytes when a stable correlation between plasma and salivary levels is proved. Used as diagnostic aids, salivary biomolecules can identify a variety of cancers, illicit and prescription drug use, hereditary disorders and hormonal irregularities.

The development of salivary diagnostics allows health professionals early disease prevention, ease biomarker monitoring and thus, provides a more predictable outcome for the patient.

\section{REFERENCES}

1. Greabu M, Battino M, Mohora M, Totan A, Didilescu A, Spinu T, et al. Saliva-a diagnostic window to the body, both in health and in disease. J Med Life. 2009;2(2):124-32.

2. Lima DP, Diniz DG, Moimaz SA, Sumida DH, Okamoto AC. Saliva: reflection of the body. Int J Infect Dis. 2010;14(3):e184-8. doi: 10.1016/j. ijid.2009.04.022.

3. Chiappin S, Antonelli G, Gatti R, De Palo EF. Saliva specimen: a new laboratory tool for diagnostic and basic investigation. Clin Chim Acta. 2007;383(1-2):30-40. doi: 10.1016/j.cca.2007.04.011

4. Martí-Álamo S, Mancheño-Franch A, MarzalGamarra C, Carlos-Fabuel L. Saliva as a diagnostic fluid. Literature review. J Clin Exp Dent. 2012;4(4):e237-43. doi: 10.4317/jced.50865.

5. Yamuna P, Muthu P. Methods of collection of saliva - a review. Int J Oral Health Dentistry. 2017;3(3):149-53. doi:10.18231/2395-499X.2017.0032.

6. Arunkumar S, Arunkumar JS, Burde KN, Shakunthala GK. Developments in diagnostic applications of saliva in oral and systemic diseases- A comprehensive review. J Sci Innov Res.2014; 3(3):372-87.

7. Meenu S, Sayanti S, Mayank B, Pragzna Y, Dharmadev B. Non-invasive diagnostic tool for pathological conditions: salivary biomarkers. Int J Pharm Biol Arch. 2014; 5(3): 1 - 12 
8. Malathi N, Mythili S, Vasanthi HR. Salivary diagnostics: A brief review. ISRN Dentistry. 2014;2014:158786.

9. Nainan OV, Xia G, Vaughan G, Margolis HS. Diagnosis of hepatitis A virus infection: a molecular approach .Clin Microbiol Rev. 2006;19(1):63-79. doi: 10.1128/CMR.19.1.63-79.2006.

10. Hermida M, Ferreiro MC, Barral S, Laredo R, Castro A, Diz Dios P. Detection of HCV RNA in saliva of patients with hepatitis $C$ virus infection by using a highly sensitive test. J Virol Methods. 2002;101(1-2):29-35.

11. Streckfus C, Bigler L. The use of soluble, salivary cerbB-2 for the detection and post-operative followup of breast cancer in women: the results of a fiveyear translational research study. Adv Dent Res. 2005;18(1):17-24. doi: 10.1177/154407370501800105.

12. Majid S, Shafi M. Saliva as a diagnostic tool: a review. IOSR J Pharm Biol Sci (IOSR-JPBS). 2014;9(1):9-14.

13. Mittal S, Bansal V, Garg S, Atreja G, Bansal S.The diagnostic role of saliva - a review. J Clin Exp Dent. 2011;3(4):e314-20. doi:10.4317/jced.3.e314.

14. Chianeh YR, Prabhu K. Biochemical markers in saliva of patients with oral squamous cell carcinoma. Asian Pac J Trop Dis. 2014; 4(1):33-40. doi: 10.1016/ S2222-1808(14)60412-6.

15. Wu CC, Chang YT, Chang KP, Liu YL, Liu HP, Lee IL, et al. Salivary auto-antibodies as noninvasive diagnostic markers of oral cavity squamous cell carcinoma. Cancer Epidemiol Biomarkers Prev. 2014;23(8):1569-78. doi: 10.1158/1055-9965. EPI-13-1269.

16. Lakshmi KR, Nelakurthi H, Kumar AS, Rudraraju A. Oral fluid-based biosensors: A novel method for rapid and noninvasive diagnosis. Indian J Dent Sci. 2017;9(1):60-6. doi: 10.4103/IJDS.IJDS_6_17.

17. Miller CS, Foley JD, Bailey AL, Campell CL, Humphries RL, Christodoulides N, et al. Current developments in salivary diagnostics. Biomark Med. 2010;4(1):171-89.

18. Adam DJ, Milne AA, Evans SM, Roulston JE, Lee AJ, Ruckley CV, et al. Serum amylase isoenzymes in patients undergoing operation for ruptured and non-ruptured abdominal aortic aneurysm. J Vasc Surg. 1999;30(2):229-35.

19. Walt DR, Blicharz TM, Hayman RB, Rissin DM, Bowden M, Siqueira WL, et al. Microsensor ar- rays for saliva diagnostics. Ann N Y Acad Sci. 2007;1098:389-400. DOI: 10.1196/annals.1384.031.

20. Arregger AL, Cardoso EM, Tumilasci O, Contreras LN. Diagnostic value of salivary cortisol in end stage renal disease. Steroids. 2008;73(1):77-82. doi: 10.1016/j.steroids.2007.09.001.

21. Malamud D, Rodriguez-Chavez IR. Saliva as a diagnostic fluid. Dental clinics of North America. 2011;55(1):159-78.

22. Kaufman E, Lamst IB. The diagnostic applications of saliva-a review. Crit Rev Oral Biol Med. 2002;13(2):197-212.

23. Hershkovich O, Nagler RM. Biochemical analysis of saliva and taste acuity evaluation in patients with burning mouth syndrome, xerostomia and/or gustatory disturbances. Arch Oral Biol 2004;49(7):51522. doi: 10.1016/j.archoralbio.2004.01.012.

24. Jyoti M, Lovleen S, Ramanpreet K, Anurag K, Anurag T. Saliva in forensics. World J Pharm Med Res. 2016,2(4):196-8.

25. Bader HI. Salivary diagnostics in medicine and dentistry: a review. Dent Today. 2011;30(8):46, 48, 50-1; quiz 52-3.

26. Casiglia J, Woo SB. A comprehensive review of oral cancer. Gen Dent. 2001;49(1):72-82.

27. Li Y, St. John MA, Zhou X, Kim Y, Sinha U, Jordan RC, et al. Salivary transcriptome diagnostics for oral cancer detection. Clin Cancer Res. 2004;10(24):8442-50. doi: 10.1158/1078-0432. CCR-04-1167.

28. Lee Y-H, Wong DT. Saliva: An emerging biofluid for early detection of diseases. A Am J Dent. 2009;22(4):241-8.

29. Babu NC, Gomes AJ. Systemic manifestations of oral diseases. J Oral Maxillofac Pathol. 2011;15(2):144-7. doi: 10.4103/0973-029X.84477.

30. Scannapieco FA. Periodontal inflammation: from gingivitis to systemic disease? Compend Contin Educ Dent. 2004;25(7 Suppl 1):16-25.

31. Loos BG, Craandijk J, Hoek FJ, Wertheim-van Dillen PM, van der Velden U. Elevation of systemic markers related to cardiovascular disease in the peripheral blood of periodontitis patients. J Periodontol. 2000; 71(10):1528-34. doi: 10.1902/ jop.2000.71.10.1528. 